\title{
Sampled-data control of hybrid systems with discrete inputs and outputs
}

\author{
M. Petreczky ${ }^{\dagger}$ P. Collins ${ }^{\ddagger}$ D.A. van Beek ${ }^{\dagger}$ J. H. van Schuppen ${ }^{\ddagger}$ J.E. $\operatorname{Rooda}^{\dagger}$ \\ ${ }^{\dagger}$ Eindhoven University of Technology, The Netherlands \\ $\{$ M.Petreczky, D.A.v.Beek, J.E.Rooda\}@tue.nl \\ ${ }^{\ddagger}$ Centrum voor Wiskunde en Informatica $(C W I)$, The Netherlands, \\ \{Pieter.Collins,J.H.van.Schuppen\}@cwi.nl
}

\begin{abstract}
We address the control synthesis of hybrid systems with discrete inputs, disturbances and outputs. The control objective is to ensure that the events of the closed-loop system belong to the language of the control requirements. The controller is sampling-based and it is representable by a finitestate machine. We formalize the control problem and provide a theoretically sound solution. The solution is based on solving a discrete-event control problem for a finite-state abstraction of the plant. We propose a specific construction for the finite-state abstraction. This construction is not based on discretizing the state-space, but rather on converting the continuous-time hybrid system to a discrete-time one based on sampling. The construction works only for a specific class of hybrid systems. We describe this class of systems and we provide an example of such a system, inspired by an industrial use-case.
\end{abstract}

Keywords: hybrid systems, discrete-event systems, symbolic control

\section{INTRODUCTION}

Motivated by applications in the area of high-tech systems, in particular control of printers, Petreczky et al. (2008b), we are interested in the following control problem. The plant is a continuous-time hybrid system which is subject to discrete disturbances and control inputs and which generates discrete outputs and internal events. The disturbances are imposed by the environment and the control inputs can be used to influence the system behavior. The desired controller can read the outputs and it generates control inputs. Furthermore, the controller should be realizable by a finite-state machine, and it is activated at equidistant sampling times with sampling rate $\Delta$. The control objective is to ensure that the sequences of internal events generated by the plant satisfy the control requirements.

Contribution We present a mathematical formulation of the control problem above. We also propose the following solution.

Step 1 Compute an abstraction (over-approximation) of the symbolic (event) behavior of the plant, such that the abstraction has a finite-state representation. This abstraction is based on transforming the original system to a discretetime one. The states of the abstraction are those states of the hybrid system which can be reached at sampling times. Under suitable assumptions, the thus obtained state-space is finite.

Step 2 Solve the related discrete-event control problem for the finite-state abstraction. The solution is a discrete-event controller representable by a Moore-automaton. Interpret the solution as a controller for the original plant.

We prove that the procedure above is theoretically sound. The discrete-event control problem of Step 2 can be solved using game theory, see Grädel et al. (2002) or, under additional assumptions, using classical supervisory control, see Petreczky et al. (2008a). We also present a procedure for constructing a finite-state abstraction. The procedure can be made effective,

1 This work was partially supported by the ITEA project Twins 05004. but it may be computationaly expensive. The finite-state abstraction can be computed only for a specific class of hybrid systems which satisfies the following properties; (1) disturbances or internal events do not influence the continuous dynamics, (2) output events do not influence the system dynamics, (3) only finitely many events are generated on any time interval, (4) the set of states reachable at sampling times is finite. For the last property we present sufficient conditions in terms of existence of a Lyapunov-like function. While these assumptions are strong, there are hybrid systems of practical relevance (see Petreczky et al. (2008b) and the example of this paper) for which they hold.

Related work To the best of our knowledge, the contribution of the paper is new. Control of hybrid systems using finitestate approximation is a classical topic, Alur et al. (2000); Gonzalez et al. (2001); Chutinan and Krogh (2003); Förstnera et al. (2002); Moor et al. (2002); Koutsoukos et al. (2000). The main difference with respect to Gonzalez et al. (2001); Chutinan and Krogh (2003); Koutsoukos et al. (2000) is the presence of partial observations, that the generation of events is not synchronous with inputs, and that the hybrid plant contains reset maps. With respect to Förstnera et al. (2002); Moor et al. (2002) the main differences are that we consider hybrid systems as opposed to continuous ones, and we address partial observations. In addition, we do not propose a general purpose finitestate abstraction, rather the proposed abstraction is intended as a vehicle for solving the specific control problem. The results of Raisch and O'Young (1995); Moor and Raisch (1999); Raisch (2000) address a problem which is quite different from the one considered in this paper. The approach of the paper resembles Alur et al. (2000); Tabuada and Pappas (2005); Fainekos et al. (2007); Belta et al. (2005). However, the abstraction notion of this paper and the problem formulation are different. The control problem of this paper is different from Philips et al. (2003). In addition, the computation of the finite-state abstraction proposed in this paper is quite different from that of the papers 
cited above. In Chutinan and Krogh (2003); Koutsoukos et al. (2000); Alur et al. (2000); Fainekos et al. (2007); Belta et al. (2005); Philips et al. (2003) the finite-state abstraction is computed by dividing the state-space of the system into regions. In Förstnera et al. (2002); Moor et al. (2002); Raisch and O'Young (1995); Moor et al. (2002); Moor and Raisch (1999); Raisch (2000), the abstraction of the system is constructed by storing the output (or state) response of the system to input sequences of finite length. In contrast, here the abstraction is obtained by sampling the hybrid system in time, not by discretizing it in space. In particular, the abstraction lives on the same state-space as the original system.

Outline of the paper In $\S 3$ we state the control problem we want to solve. The reduction of the hybrid problem to a discrete-event one is discussed in $\S 4$. In $\S 5$ the class of hybrid systems of interest is defined and the computation of a finitestate abstraction of the hybrid plant is discussed. In $\S 6$, as an illustration, we present an example.

\section{PRELIMINARIES}

General notation We use the standard notation and terminology from automata theory Eilenberg (1974). $\mathbb{N}$ is the set of natural numbers including zero. If $\Sigma$ is a finite alphabet, then $\Sigma^{*}$ denotes the set of finite strings (words) on $\Sigma$. The empty word is denoted by $\epsilon$. An infinite word over $\Sigma$ is an infinite sequence $w=a_{1} a_{2} \cdots a_{k} \cdots$ with $a_{i} \in \Sigma, i \in \mathbb{N}$. The set of infinite words is denoted by $\Sigma^{\omega}$. The length of a (in)finite word is denoted by $|w|$; if $w$ is an infinite word, then $|w|=+\infty$. For any (in)finite word $w$, and for any $i \in \mathbb{N}$ (in case $w$ is finite word, for any $0 \leq i \leq|w|), w_{1: i}$ denotes the finite word formed by the first $i$ letters of $w$, i.e. $w_{1: i}=a_{1} a_{2} \cdots a_{i}$. If $i=0$, then $w_{1: i}$ is the empty word $\epsilon$. The set of non-negative reals is $\mathbb{R}_{+}$.

Moore-automata A Moore-automaton (Eilenberg (1974)) is a tuple $A=\left(Q, I, Y, \delta, \lambda, q_{0}\right)$ where $Q$ is the finite state-space, $I$ is the input alphabet, $Y$ is the output alphabet, $\delta: Q \times I \rightarrow Q$ is the state-transition map, $\lambda: Q \rightarrow Y$ is the readout map, and $q_{0} \in Q$ is the initial state. The Moore-automaton $A$ is a realization of a map $\phi: I^{*} \rightarrow Y$, if for all $w=u_{1} u_{2} \cdots u_{k} \in$ $I^{*}, k \geq 0$ and $u_{1}, u_{2}, \ldots, u_{k} \in I, \phi(w)=\lambda\left(q_{k}\right)$ where $q_{i}=\delta\left(q_{i-1}, u_{i}\right)$ for all $i=1,2, \ldots, k$.

Monoid automata Recall from Berstel (1979); Eilenberg (1974) that a monoid $M$ is a semi-group with a unit element. A finite-state automaton on a monoid $M$, abbreviated as DFA, is a tuple $T=\left(Q, M, E, F, q_{0}\right)$ where $Q$ is a finite set of states, $M$ is the monoid of inputs, $E \subseteq Q \times M \times Q$ is a state-transition relation, where $E$ is a finite set, $F \subseteq Q$ is the set of accepting states, $q_{0} \in Q$ is the initial state. An element $m \in M$ is accepted by $T$ if there exists $m_{i} \in M_{i}$ and $q_{i} \in Q$, $i=1,2, \ldots, k, k \geq 0$ such that $\left(q_{i}, m_{i+1}, q_{i+1}\right) \in E$ for $i=0,1, \ldots, k-1, q_{k} \in F$ and $m=m_{1} m_{2} \cdots m_{k}$. The set $L \subseteq M$ is recognized by $T$, denoted by $L(T)$, if $L$ consists of precisely those elements $m \in M$ which are accepted by $T$.

Sequential input-output maps will be used to model the discrete-event abstractions of hybrid systems. The concepts below are discussed in more detail in Petreczky et al. (2008a).

Definition 1. A multi-valued map $R: \Sigma^{*} \rightarrow 2^{X^{*} \times Y^{*}}$ is called a sequential input-output map, if

(1) $R(\epsilon)=(\epsilon, \epsilon)$, and for all $s \in \Sigma^{*}, R(s)$ is a nonempty set. Furthermore, $R$ is length-preserving in its $X$-valued component, i.e. if $(\underline{x}, y) \in R(s)$, with $\underline{x} \in X^{*}$ and $y \in Y^{*}$, then the length of $s$ and $\underline{x}$ are the same, i.e. $|s|=|\underline{x}|$,

(2) $R$ is prefix preserving, i.e. for each word $s \in \Sigma^{*}$ and letter $a \in \Sigma$, if $(\underline{x}, \underline{y}) \in R(s a)$, then there exist $x \in X$ and $y \in Y^{*}$, $\underline{\hat{x}} \in X^{*}, \underline{\hat{y}} \in Y^{*}$ such that $\underline{x}=\underline{\hat{x}} x, \underline{y}=\underline{\hat{y}} y$ and $(\underline{\hat{x}}, \underline{\hat{y}}) \in R(s)$, (3) $R$ is non-blocking, i.e. for each $s \in \Sigma^{*}, a \in \bar{\Sigma},(\underline{x}, \underline{y}) \in$ $R(s),(\underline{x} x, \underline{y} y) \in R(s a)$ for some $x \in X, y \in Y^{*}$.

Definition 2. A DFA $T=\left(Q, M, E, F, q_{0}\right)$ defined over the monoid $M=\Sigma^{*} \times X^{*} \times Y^{*}$ is called a quasi-sequential transducer, if (1) $F=Q$, i.e. all states are accepting, (2) the state-transition relation $E$ is a partial map $E: Q \times \Sigma \times X \times$ $Y^{*} \rightarrow Q$, (3) for each state $q \in Q$ and letter $a \in \Sigma$ there exist $x \in X$ and $y \in Y^{*}$ such that $E(q, u, x, y)$ is defined.

Definition 3. The sequential input-output map $R: \Sigma^{*} \rightarrow$ $2^{X^{*} \times Y^{*}}$ is quasi-recognizable, if there exists a quasi-sequential transducer which recognizes the graph of $R$, i.e. which recognizes the set $\left\{(\underline{u}, \underline{x}, \underline{y}) \in \Sigma^{*} \times X^{*} \times Y^{*} \mid(\underline{x}, \underline{y}) \in R(\underline{u})\right\}$.

\section{CONTROL PROBLEM}

The plant of interest is a hybrid system which reacts to discretevalued control inputs and disturbances, and generates discretevalued outputs and internal events. We view the inputs and outputs as discrete events. Thus, the control inputs are events generated by a potential controller, the disturbances are events generated by the environment. The outputs and internal events are events generated by the plant. The only difference between outputs and internal events is that outputs are visible (i.e. detectable by sensors), while internal events are not.

Notation 1. (Plant and events). We denote the plant by $H$. We denote by $E_{c}$ the set of control inputs, $E_{d}$ the set of disturbances, $E_{o}$ the set of outputs, $E_{i}$ the set of internal events. We assume that $E_{c}, E_{d}, E_{o}, E_{i}$ are finite sets.

In order to define the input-output behavior of the plant formally, we need the following notion.

Definition 4. Let $E$ be a finite set and let $\perp \notin E$. Consider a (in)finite timed sequence of elements of $E$.

$$
s=\left(e_{1}, t_{1}\right)\left(e_{2}, t_{2}\right) \cdots\left(e_{k}, t_{k}\right) \cdots
$$

where $0 \leq t_{1}<t_{1}<t_{2}<\cdots, e_{i+1} \in E, t_{i+1} \in \mathbb{R}_{+}$for $i \in \mathbb{N}, i<|s|$. Here $|s|$ is the length of $s$, and $|s|=+\infty$ if $s$ is an infinite sequence. If $|s|=+\infty$, then we assume that $\sup _{i \in \mathbb{N}} t_{i+1}=+\infty$. We can identify $s$ with a map

$$
g: \mathbb{R}_{+} \ni t \mapsto\left\{\begin{array}{ll}
e_{i+1} \in E & \text { if } t=t_{i+1} \\
\perp & \text { otherwise }
\end{array} \text { for some } i \in \mathbb{N}\right.
$$

The map $g$ above, is called a time-event map. The set of all such maps is denoted by $\mathcal{P}_{E}$. Denote the sequence of elements of $E$ induced by $g$ by $\mathbf{U T}(g)=e_{1} e_{2} \cdots e_{k} \cdots \in E^{*} \cup E^{\omega}$.

I.e., the timed-event function $g$ takes values in the event set $E$ at isolated time instances, and the value $\perp$ encodes the absence of events at a certain time instance. By applying the above definition to $E \in\left\{E_{c}, E_{d}, E_{o}, E_{i}\right\}$, we obtain the sets $\mathcal{P}_{E_{c}}, \mathcal{P}_{E_{d}}, \mathcal{P}_{E_{o}}, \mathcal{P}_{E_{i}}$ describing the time signals with values in inputs, disturbances, outputs and internal events respectively.

Definition 5. (Input-output map of the plant). The input-output map of $H$ is a causal ${ }^{2}$ map $v_{H}: \mathcal{P}_{E_{c}} \times \mathcal{P}_{E_{d}} \rightarrow \mathcal{P}_{E_{o}} \times \mathcal{P}_{E_{i}}$. Definition 6. A hybrid controller is a map $\mathcal{C}: \mathcal{P}_{E_{o}} \rightarrow \mathcal{P}_{E_{c}}$.

We study controllers which have a finite-state representation and are activated at fixed sampling rate $\Delta>0$. The controller can only detect the set of outputs which occurred in a sampling interval. The formal definition is as follows.

2 By causality of $v_{H}$ we mean that the response of $v_{H}$ depends only on the past inputs and on the past and present disturbances, i.e. for any $u_{i} \in \mathcal{P}_{E_{c}}$ $d_{i} \in \mathcal{P}_{E_{d}},\left(o_{i}, \hat{o}_{i}\right)=v_{H}\left(u_{i}, d_{i}\right), i=1,2$, if $\left.d_{1}\right|_{[0, t]}=\left.d_{2}\right|_{[0, t]},\left.u_{1}\right|_{[0, t)}=$ $\left.u_{2}\right|_{[0, t)}$ then $o_{1}(t)=o_{2}(t)$ and $\hat{o}_{1}(t)=\hat{o}_{2}(t)$, for all $t \in \mathbb{R}_{+}$. 


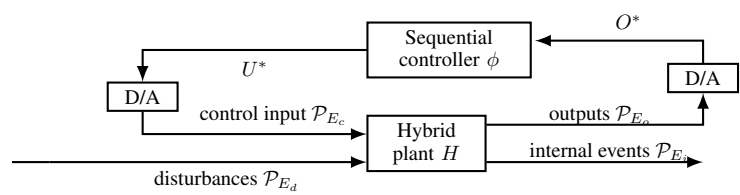

Fig. 1. Control architecture

Definition 7. Let $U=E_{c} \cup\{\perp\}$ be the sampled input set, let $O=2^{E_{o}}$ be the sampled output set. A sequential controller is a map $\phi: O^{*} \rightarrow U$ which has a Moore-automaton realization.

The desired hybrid controller is then a hybrid controller associated with a sequential controller and it is defined as follows.

Definition 8. (Sampling-based controller). For a sequential controller $\phi$ let the hybrid controller $\mathcal{C}_{\phi}: \mathcal{P}_{E_{o}} \rightarrow \mathcal{P}_{E_{c}}$ associated with $\phi$ be such that for all $o \in \mathcal{P}_{E_{o}}$, and for all $t \in \mathbb{R}_{+}$,

$\mathcal{C}_{\phi}(o)(t)=\left\{\begin{aligned} \phi\left(S_{1} S_{2} \cdots S_{k+1}\right) & \text { if } t=(k+1) \Delta \text { for a } k \in \mathbb{N} \\ \phi(\epsilon) & \text { if } t=0 \\ \perp & \text { otherwise }\end{aligned}\right.$

where $S_{k+1}=o((k \Delta,(k+1) \Delta]) \cap E_{o}$ for all $k \in \mathbb{N}$.

Next, we define the relevant aspects of the closed-loop behavior of the system. First, in order to avoid technical difficulties, we restrict attention to disturbances where at most a fixed $\mu$ number of disturbance events occurs within a sampling interval.

Definition 9. Let $\mu \in \mathbb{N}$ be the upper bound on the number of disturbances in a sampling interval. Denote by $\mathcal{P}_{E_{d}, \mu}^{\Delta}$ the set of functions $g \in \mathcal{P}_{E_{d}}$ such that on any interval $(i \Delta,(i+1) \Delta]$, $i \in \mathbb{N}$ the number of events of $g$ is not greater than $\mu$, i.e. $\operatorname{card}\left\{e=g(s) \in E_{d} \mid s \in(i \Delta,(i+1) \Delta]\right\} \leq \mu$.

Definition 10. Let $\phi$ be a sequential controller and let $\mathcal{C}_{\phi}$ be the asscociated hybrid controller. The closed-loop language $L\left(H / \mathcal{C}_{\phi}\right)$ be the set of (in)finite words of the form $\mathbf{U T}(\hat{o}) \in$ $E_{i}^{*} \cup E_{i}^{\omega}$, where $\hat{o} \in \mathcal{P}_{E_{i}}$ and there exist $u \in \mathcal{P}_{E_{c}}, d \in \mathcal{P}_{E_{d}, \mu}^{\Delta}$, and $o \in \mathcal{P}_{E_{o}}$ such that $(o, \hat{o})=v_{H}(u, d)$ and $u=\mathcal{C}_{\phi}(o)$.

That is, $L\left(H / \mathcal{C}_{\phi}\right)$ is the set of sequences of internal events generated by the interconnection of the plant $H$ with the controller $\mathcal{C}_{\phi}$. The control problem of interest can be stated as follows.

Problem 1. (Sampled-data control). For a specification language $K \subseteq E_{i}^{*} \cup E_{i}^{\omega}$, find a sequential controller $\phi$ such that for the closed-loop language satisfies $L\left(H / \mathcal{C}_{\phi}\right) \subseteq K$.

Note that the results of the paper can easily be extended so that the specification language includes events from $E_{c} \cup E_{d} \cup E_{o}$.

\section{SOLUTION OF THE HYBRID CONTROL PROBLEM}

In this section we present the solution of Problem 1. The main idea is to reduce Problem 1 to a discrete-event control problem. To this end, we model the sampled-data behavior of the plant as a discrete-event system $R_{H}$, which reacts to sampled inputs from $U$ and sampled disturbances from $D$ (to be defined below) and generates sampled outputs from $O$ and sequences internal events. In order to define $R_{H}$, we need the following.

Definition 11. The set sampled disturbances of $R_{H}$ is defined as $D=\bigcup_{k=0}^{\mu} E_{d}^{k}$, where $\mu$ is as in Definition 9.

That is, $D$ is the set of all words over $E_{d}$ of length at most $\mu$. Notation 2. Let $g \in \mathcal{P}_{E}$ be a time-event function. For all $t \in \mathbb{R}^{+}$, let $\mathbf{U T}(g, t) \in E^{*}$, be the sequence of events of $g$ up to $t$, i.e. $\mathbf{U T}(g, t)=\mathbf{U T}\left(g^{t}\right)$, where $g^{t} \in \mathcal{P}_{E}$ is such that $g^{t}(s)=g(s)$ if $s \leq t$ and $g^{t}(s)=\perp$ for all $s>t$

Definition 12. The sequential input-output map $R_{H}$ of $H$ is the map $R_{H}:(U \times D)^{*} \rightarrow 2^{O^{*} \times E_{o}^{*}}$ defined as follows.
$R_{H}(\epsilon)=\{(\epsilon, \epsilon)\}$ and for each sequence of sampled inputs $u_{1}, u_{2}, \ldots, u_{k} \in U$ and disturbances $d_{1}, d_{2}, \cdots d_{k} \in D, k \geq 0$, $\left(o_{1} o_{2} \cdots o_{k}, \underline{\hat{o}}\right) \in R_{H}\left(\left(u_{1}, d_{1}\right)\left(u_{2}, d_{2}\right) \cdots\left(u_{k}, d_{k}\right)\right)$

for some $o_{1}, o_{2}, \ldots, o_{k} \in O$, and $\underline{\hat{o}} \in E_{i}^{*}$, if there exist $g \in \mathcal{P}_{E_{d}}, o \in \mathcal{P}_{E_{o}}, \hat{o} \in \mathcal{P}_{E_{i}}$ such that $(o, \hat{o})=v_{H}(u, g)$,

$$
\forall t \in \mathbb{R}_{+}: u(t)= \begin{cases}u_{i} & \text { if } t=(i-1) \Delta \text { for } i=1,2, \ldots, k \\ \perp & \text { otherwise }\end{cases}
$$

$\underline{\hat{o}}=\mathbf{U T}(\hat{o}, k \Delta)$, and $o_{i}=o(((i-1) \Delta, i \Delta]), d_{i}=\mathbf{U T}\left(g_{i}, \Delta\right)$, where $g_{i}(t)=g(t+(i-1) \Delta), \forall t \in \mathbb{R}_{+}$, for all $i=1,2, \ldots, k$.

$R_{H}$ is a sequential input-output map of Definition 13. Intuitively, $R_{H}$ is the result of composing $H$ with the interfaces converting time-event functions from $\mathcal{P}_{E_{o}}, \mathcal{P}_{E_{i}}, \mathcal{P}_{E_{d}, \mu}^{\Delta}$, to sequences in $O^{*}, E_{i}^{*}$ and $D^{*}$, and with the interface which converts sequences from $U^{*}$ to maps $\mathcal{P}_{E_{c}}$.

In order to solve Problem 1 , we can view $R_{H}$ as a discreteevent plant, and solve a discrete-event control problem for $R_{H}$ as a plant and $K$ as a requirement. The discrete-event control problem is as follows.

Definition 13. A discrete-event plant is a sequential inputoutput map $R:(U \times D)^{*} \rightarrow 2^{O^{*} \times E_{i}^{*}}$.

Definition 14. The closed-loop language $L(R / \phi) \subseteq E_{i}^{*} \cup$ $E_{i}^{\omega}$ of the interconnection of $R$ with the sequential controller $\phi: O^{*} \rightarrow U$ is the set of all words $\underline{\hat{o}} \in E_{i}^{*} \cup E_{i}^{\omega}$ for which there exist $d_{i} \in D, o_{i} \in O, u_{i} \in U, \bar{i}=1,2, \ldots$, and indices $k_{1} \leq k_{2} \leq \cdots k_{i} \leq \operatorname{such}$ that $\sup _{i \in \mathbb{N}} k_{i+1}=|\underline{\hat{o}}|$, and $\forall i \in \mathbb{N}$,

$\left(o_{1} o_{2} \cdots o_{i+1}, \underline{\hat{o}}_{1: k_{i+1}}\right) \in R\left(\left(u_{1}, d_{1}\right)\left(u_{2}, d_{2}\right) \cdots\left(u_{i+1}, d_{i+1}\right)\right)$ $u_{i+1}=\phi\left(o_{1} o_{2} \cdots o_{i}\right)$ if $i>0$, and $u_{1}=\phi(\epsilon)$

Problem 2. (Discrete control problem). For the plant $R$, and for the control requirements $K \subseteq E_{i}^{*} \cup E_{i}^{\omega}$, find a sequential controller $\phi$ such that $L(R / \phi) \subseteq K$ holds.

Theorem 1. (Hybrid vs. discrete control). If $\phi$ is a sequential controller, then $L\left(H / \mathcal{C}_{\phi}\right) \subseteq L\left(R_{H} / \phi\right)$. Hence, if $\phi$ solves Problem 2 for $R=R_{H}$, and $K \subseteq E_{i}^{*} \cup E_{i}^{\omega}$, then the associated hybrid controller $\mathcal{C}_{\phi}$ solves Problem 1 for $H$ and $K$.

For more details on the solution of Problem 2, see Petreczky et al. (2008a). A necessary condition for effective solution of Problem 2 is that $R$ is quasi-recognizable, i.e. it is recognized by a quasi-sequential transducer. However, $R_{H}$ need not be quasi-recognizable. The remedy is to solve Problem 2 not for $R_{H}$ but for an quasi-recognizable abstraction of $R_{H}$. The construction of the latter is discussed in $\S 5$.

Definition 15. (Abstraction). The sequential input-output map $R$ is an abstraction of the map $R_{H}$ if for all $s \in(U \times D)^{*}$, the inclusion $R_{H}(s) \subseteq R(s)$ holds.

Theorem 2. Assume that $R$ is an abstraction of $R_{H}$. Then for any sequential controller $\phi, L\left(R_{H} / \phi\right) \subseteq L(R / \phi)$. Hence, if $\phi$ solves Problem 2 for $R$, then $\phi$ solves Problem 2 for $R_{H}$.

Hence, in order to solve Problem 1, we have to compute a quasirecognizable abstraction $R$ of $R_{H}$ as described in $\S 5$, and then solve the discrete control problem Problem 2 for $R$.

\section{FINITE-STATE ABSTRACTION OF $R_{H}$}

First we define the hybrid systems of interest.

Definition 16. (Hybrid systems of interest). A discrete i/o hybrid system $H$ is a tuple

$$
\left(S_{H}, \delta, \lambda_{i}, \lambda_{o},\left\{f_{q}, R_{u, q}, \Phi_{q, e}\right\}_{q \in Q, u \in E_{c}, e \in E_{i} \cup E_{o}}, h_{0}\right)
$$


- Events $E_{d}$ is the set of disturbances, $E_{c}$ is the set of control inputs, $E_{o}$ is the set of outputs, $E_{i}$ is the set of internal events, and $E_{c}, E_{d}, E_{i}, E_{o}$ are finite sets.

- State-space $S_{H}=Q \times \mathcal{X}$ is the state-space of $H$. Here $Q=Q_{c} \times Q_{d}$ is the discrete state-space of $H, Q_{c}, Q_{d}$ are finite sets. The set $\mathcal{X} \subseteq \mathbb{R}^{n}$ is the continuous state-space, $\mathcal{X}$ is a closed set with non-empty interior int $\mathcal{X} \neq \emptyset$.

- Discrete-state transition is determined by the transition functions $\delta_{c}: Q \times E_{c} \rightarrow Q_{c}, \delta_{d}: Q \times\left(E_{d} \cup E_{i}\right) \rightarrow Q_{d}$.

- Continuous dynamics is determined by vector fields $f_{q^{c}}$ : $\mathbb{R}^{n} \rightarrow \mathbb{R}^{n}, q \in Q_{c}$, and reset maps $R_{u, q}: \mathcal{X} \rightarrow \mathcal{X}, q \in Q$ and $u \in E_{c}$. The vector fields $f_{q^{c}}, q^{c} \in Q_{c}$ are continuous and globally Lipschitz.

- Event generation is determined by guards $\Phi_{q, e} \subseteq \mathcal{X}$, $q \in Q, e \in E_{o} \cup E_{i}$, and by discrete partial readout maps $\lambda_{o}: Q \times E_{d} \rightarrow E_{o}, \lambda_{i}: Q \times E_{d} \rightarrow E_{i}$.

- $h_{0}=\left(q_{0}^{c}, q_{0}^{d}, x_{0}\right) \in S_{H}$ is the initial state of the system. Moreover, $x_{0} \in \operatorname{int} \mathcal{X}$, i.e. $x_{0}$ is in the interior of $\mathcal{X}$.

The system $H$ is a hybrid system in the sense of van der Schaft and Schumacher (2000), subject to the following restrictions. Consider a discrete state $q=\left(q^{c}, q^{d}\right) \in Q$ If an event $u \in E_{c}$ arrives, then the $Q_{c}$-valued state component changes to $\delta_{c}(q, u)$. If $e \in E_{d} \cup E_{i}$ occurs, then the $Q_{d}$-valued discrete state component changes to $\delta_{d}(q, e)$. For an event from $E_{o}$ the discrete state does not change. The continuous dynamics in $q$ is determined by the differential equation $\dot{x}=f_{q^{c}}(x)$, as long as the continuous state is in the interior of $\mathcal{X}$. As soon as the continuous state reaches the boundary, it will change only if a reset map is applied. The reset maps for an event $u \in E_{c}$ are specified by $R_{u, q}$. For all the events from $E_{d} \cup E_{o} \cup E_{i}$, the reset maps are the identity. An event $e \in E_{o} \cup E_{i}$ is generated either if the continuous state crosses a guard set $\Phi_{q, e}$ or when an event from $d \in E_{d}$ arrives. In the latter case, $e=\lambda_{i}(q, d)$ or $e=\lambda_{o}(q, d)$. Events from $E_{c} \cup E_{d}$ are generated by the controller/environment. For the formal definition of the state evolution, we need the following.

Definition 17. (Flow of $f_{q^{c}}$ ). For any time $t \in \mathbb{R}_{+}$and for any $q^{c} \in Q_{c}$ define the flow $f_{q^{c}}^{t}: \mathcal{X} \rightarrow \mathcal{X}$ of $f_{q^{c}}$ as follows. Consider the solution of the differential equation $\dot{z}=f_{q^{c}}(z)$ with the inital state $z(0)=z_{0}$. Define

$$
f_{q^{c}}^{t}\left(z_{0}\right)= \begin{cases}z(t) & \text { if } t<\beta\left(q^{c}, z_{0}\right) \\ z\left(\beta\left(q^{c}, z_{0}\right)\right. & \text { if } \beta\left(q^{c}, z_{o}\right) \leq t<+\infty\end{cases}
$$

where $\beta=\beta\left(q^{c}, z_{0}\right) \in[0,+\infty]$ is such that for all $t \in[0, \beta)$, $z(t) \in$ int $\mathcal{X}$ and if $\beta<+\infty$, then $z(\beta) \in \partial X$, i.e. $z(\beta)$ belongs to the boundary of $\mathcal{X}$.

Notice that for any $z_{0} \in \partial X, f_{q^{c}}^{t}\left(z_{0}\right)=z_{0}$, i.e. the continuous state evolution stops on the boundary of $\mathcal{X}$. The following assumptions will be used in the rest of the paper.

Assumption 1. A.1. For any $\Sigma \in\left\{E_{o}, E_{i}\right\}$ and $q \in Q, \forall e_{1} \neq$ $e_{2} \in \Sigma: \Phi_{q, e_{1}} \cap \Phi_{q, e_{2}}=\emptyset$.

A.2. For each $q=\left(q^{c}, q^{d}\right) \in Q=Q_{c} \times Q_{d}, e \in E_{o} \cup E_{i}$ there exist smooth maps $h_{q, e}: \mathbb{R}^{n} \rightarrow \mathbb{R}$, such that

$$
\begin{aligned}
& \Phi_{q, e} \subseteq\left\{x \in \operatorname{int} \mathcal{X} \mid h_{q, e}(x)=0\right\}, \text { and } \\
& \text { if } \Phi_{q, e} \neq \emptyset, \text { then } \forall x \in \mathbb{R}^{n}: \operatorname{grad}\left(h_{q, e}\right)(x) f_{q^{c}}(x)>0 .
\end{aligned}
$$

A.3. For any $q \in Q, d \in E_{d}, \lambda_{i}(q, d)$ is defined. Moreover, if $e=\lambda_{i}(q, d)$, then for any $\hat{q} \in Q, \Phi_{\hat{q}, e}=\emptyset$.

Assumption A.1 ensures that at most one output and at most one internal event is generated at any time instance. Assumption A. 2 requires each guard set to be a subset of a hyper-surface. In addition, the vector field associated with the discrete state has to be transversal with respect to the hyper-surface. This is a strong condition, but we believe it will be satisfied for a fairly large class of systems, for instance models of production systems and paper processing machines such as printers. Assumption A.2 ensures that only a finite number of outputs or internal events are generated on any finite time interval. Assumption A.3 allows to recognize whether an internal event is generated by a discrete readout map or by crossing a guard. Next, we define the state and output evolution of $H$.

Definition 18. For any state $h=\left(q_{h}, x_{h}\right), q_{h}=\left(q_{h}^{c}, q_{h}^{d}\right)$, input $u \in \mathcal{P}_{E_{c}}$ and disturbance $d \in \mathcal{P}_{E_{d}}$, the state-trajectory is a map

$$
\xi_{H}(h, u, d): \mathbb{R}_{+} \ni t \mapsto(q(t), x(t)) \in S_{H}
$$

where $q(t)=\left(q^{c}(t), q^{d}(t)\right) \in Q, q^{d}(0)=q_{h}^{d}, q^{c}(0)=q_{h}^{c}$ and $x(0)=x_{h}$ if $u(0)=\perp, q^{c}(0)=\delta_{c}\left(q_{h}, u(0)\right)$ and $x(0)=R_{u(0), q_{h}}\left(x_{h}\right)$ if $u(0) \in E_{c}$, and $\forall t \in \mathbb{R}_{+}, t>0$

$q^{c}(t)= \begin{cases}\delta_{c}\left(q\left(t^{-}\right), u\right) & \text { if } u=u(t) \in E_{c} \\ q^{c}(t-r) & \text { if } \exists r>0: u((t-r, t])=\{\perp\}\end{cases}$

$q^{d}(t)= \begin{cases}q^{d}(t)=\delta_{d}\left(q\left(t^{-}\right), e\right) & \text { if } d(t)=e \in E_{d}, \text { or } d(t)=\perp \\ & \text { and } x\left(t^{-}\right) \in \Phi_{q\left(t^{-}\right), e}, e \in E_{i} \\ q^{d}(t)=q^{d}(t-r) & \text { if } \exists r>0: \forall s \in(t-r, t]: \\ & d(s)=\perp, u(s)=\perp \text { and } \\ & x(s) \notin \bigcup_{e \in E_{i}} \Phi_{q(t-r), e}\end{cases}$

$x(t)= \begin{cases}R_{u, q\left(t^{-}\right)}\left(x\left(t^{-}\right)\right) & \text {if } u(t)=u \in E_{c} \\ f_{q^{c}(t)}^{r}(x(t-r)) & \text { if } \exists r>0: u((t-r, t])=\{\perp\}\end{cases}$

Here $q\left(t^{-}\right), x\left(t^{-}\right)$are the left-hand side limits at $t$ of $q(t), x(t)$. Definition 19. The input-output map of $H$ induced by state $h \in S_{H}$ is defined as

$$
v_{H, h}: \mathcal{P}_{E_{c}} \times \mathcal{P}_{E_{e}} \ni(u, d) \mapsto(o, \hat{o}) \in \mathcal{P}_{E_{o}} \times \mathcal{P}_{E_{i}}
$$

where $o(0)=\perp, \hat{o}(0)=\perp$ and for $t>0$,

$$
\begin{gathered}
o(t)=\left\{\begin{aligned}
e \in E_{o} & \text { if } x\left(t^{-}\right) \in \Phi_{q\left(t^{-}\right), e} \text { and } d(t)=\perp, \\
\lambda_{o}\left(q\left(t^{-}\right), d(t)\right) & \text { if } d(t) \in E_{d}, \\
\text { and } \lambda_{o}\left(q\left(t^{-}\right), d(t)\right) \text { is defined } & \text { otherwise }
\end{aligned}\right. \\
\hat{o}(t)=\left\{\begin{aligned}
e \in E_{i} & \text { if } x\left(t^{-}\right) \in \Phi_{q\left(t^{-}\right), e} \text { and } d(t)=\perp, \\
\lambda_{i}\left(q\left(t^{-}\right), d(t)\right) & \text { if } d(t) \in E_{d} \\
\perp & \text { otherwise }
\end{aligned}\right.
\end{gathered}
$$

where $\xi_{H}(h, u, d)(t)=(q(t), x(t))$. We denote by $v_{H}$ the input-output map $v_{H, h_{0}}$ induced by the initial state $h_{0}$ of $H$.

Informally, if there are no disturbances, then an output or internal event is generated if the continuous state crosses a guard. If a disturbance arrives, then an output (resp. internal event) is generated according to the readout map $\lambda_{o}$ (resp. $\lambda_{i}$ ).

Construction of a finite-state abstraction of $R_{H}$ Next, we present the definition of the quasi-sequential transducer, which recognizes an abstraction of $R_{H}$. In the sequel $H$ is a hybrid system from Definition 16 satisfying Assumption A.1- A.3.

Definition 20. Let $\mathcal{R}(H)=\bigcup_{i=0}^{\infty} Q \times H_{i}$ be such that

$H_{0}=\left\{x_{0}\right\}$ and $H_{i+1}=H_{i} \cup\left\{f_{q^{c}}^{\Delta}(x), f_{q^{c}}^{\Delta}\left(R_{u, s}(x)\right) \mid x \in H_{i}\right.$, $\left.q^{c} \in Q_{c}, s \in Q, u \in E_{c}\right\}, \forall i \in \mathbb{N}$

where $x_{0}$ is the continuous component of the initial state of $H$. Assumption 2. In the sequel we assume that $\mathcal{R}(H)$ is finite.

$\mathcal{R}(H)$ will be the state-space of the to be constructed abstraction. Later on we formulate conditions for finiteness of $\mathcal{R}(H)$. The main idea behind the construction of the sampledtime abstraction is that it is enough to look at states which are 
reached at sampling times, i.e. at a subset of elements of $\mathcal{R}(H)$. Moreover, the events generated in a sampling interval can be estimated by using the sampled state.

Definition 21. For any $q=\left(q^{c}, q^{d}\right) \in Q$ and $e \in E_{i} \cup E_{o}$, the guard abstraction predicate $P_{q, e} \subseteq \mathcal{X}$ is either $P_{q, e}=\emptyset$, if $e=\lambda(q, d)$ for some $d \in E_{d}$, or

$$
P_{q, e}=\left\{x \in \mathcal{X} \mid h_{q, e}(x) \leq 0 \text { and } h_{q, e}\left(f_{q^{c}}^{\Delta}(x)\right) \geq 0\right\}
$$

Informally, $P_{q, e}$ contains those continuous states, started from which the guard corresponding to $e$ is crossed within $\Delta$ time.

Definition 22. Let $\mathcal{P}=\left\{P_{q, e}\right\}_{q \in Q, e \in E_{i} \cup E_{o}}$ the collection of sets from Definition 21. Define the finite-state abstraction $H_{\Delta}$ as a quasi-sequential transducer

$$
H_{\Delta}=\left(\mathcal{R}(H),(U \times D)^{*} \times O^{*} \times E_{i}^{*}, E, \mathcal{R}(H), h_{0}\right) \text { where }
$$

Initial state $h_{0}=\left(q_{0}^{c}, q_{0}^{d}, x_{0}\right)$ of $H_{\Delta}$ coincides with that of $H$. State transition map $E: \mathcal{R}(H) \times(U \times D) \times O \times E_{i}^{*} \rightarrow \mathcal{R}(H)$ is defined as follows. For each $u \in U, d \in D, o \in O$ and $\hat{o} \in$ $E_{i}^{*}, E\left(h_{1}, u, d, o, \hat{o}\right)$ is defined and $E\left(h_{1}, u, d, o, \hat{o}\right)=h_{2}$ if and only if $h_{i}=\left(q_{i}, x_{i}\right) \in \mathcal{R}(H)$ where $q_{i}=\left(q_{i}^{c}, q_{i}^{d}\right) \in Q_{c} \times Q_{d}$ and $x_{i} \in \mathcal{X}, i=1,2$, and the following holds.

(1) The state components $q_{2}^{c}$ and $x_{2}$ are defined as follows.

$$
q_{2}^{c}=\delta_{c}\left(q_{1}, u\right) \text { and } x_{2}=f_{q_{2}^{c}}^{\Delta}\left(R_{u, q_{1}}\left(x_{1}\right)\right)
$$

Here, for $u=\perp, \delta_{c}\left(q_{1}, \perp\right)=q_{1}^{c}$ and $R_{\perp, q_{1}}\left(x_{1}\right)=x_{1}$ (2) Assume that $d=e_{1} e_{2} \cdots e_{k}, 0 \leq k \leq \mu, e_{1}, e_{2}, \ldots, e_{k} \in$ $E_{d}$. Then the sequence $\hat{o}$ is of the form $\hat{o}=z_{1} z_{2} \cdots z_{l}$, where $k \leq l \leq\left|Q_{d}\right|\left|E_{i}\right|+k$ and $z_{1}, z_{2}, \ldots, z_{l} \in E_{i}$ and the following holds. There exists a set of indices $I=\left\{i_{1}, i_{2}, \ldots, i_{k}\right\} \subseteq$ $\{1,2, \ldots, l\}, i_{1}<i_{2}<\cdots<i_{k}$ and discrete states $s_{i} \in \bar{Q}$, $i=0,1, \ldots, l$ such that $s_{0}=\left(q_{2}^{c}, q_{1}^{d}\right), s_{l}=q_{2}$ and for all $i=1,2, \ldots, l$

$$
s_{i}=\left\{\begin{array}{cc}
\left(q_{2}^{c}, \delta_{d}\left(s_{i-1}, z_{i}\right)\right) & \text { if } R_{u, q_{1}}\left(x_{1}\right) \in P_{s_{i-1}, z_{i}} \text { and } i \notin I \\
\left(q_{2}^{c}, \delta_{d}\left(s_{i-1}, e_{r}\right)\right) & \text { if } i=i_{r} \text { and } z_{i}=\lambda_{i}\left(s_{i-1}, e_{r}\right) \\
& \text { for some } r=1,2, \ldots, k,
\end{array}\right.
$$

(3) The output $o \subseteq E_{o}$ is a subset of $E_{o}$ such that for any $e \in o$,

$$
\begin{aligned}
& R_{u, q_{1}}\left(x_{1}\right) \in P_{s_{i}, e} \text { for some } i \in\{1,2, \ldots, l\} \backslash I, \text { or } \\
& \lambda_{o}\left(s_{i_{r}-1}, e_{r}\right)=e \text { for some } r=1,2, \ldots, k
\end{aligned}
$$

Intuition The states of $H_{\Delta}$ are those states of $H$ which can be reached from $h_{0}$ at sampling times. By assumption, this set is finite. A state transition of $H_{\Delta}$ associated with a discrete input $u$, disturbance $d \in D$, output $o \in O$ and sequence of internal events $\hat{o} \in E_{i}^{*}$ is obtained as follows. If the current state of $H_{\Delta}$ is $h_{1}$ then the new state $h_{2}$,is the state of $H$ reachable from $h_{1}$ in time $\Delta$, under the following conditions; (1) $H$ receives input event $u$ at time 0 , and no input after that, (2) $H$ receives a disturbance $g$, such that the sequence of events of $g$ on $(0, \Delta]$ is $d,(3) \hat{o}$ is the sequence of internal events generated by $H$ while moving from $h_{1}$ to $h_{2}$, (4) $o$ is the set of outputs generated by $H$ while moving from state $h_{1}$ to $h_{2}$. Condition (1) and the fact that the $Q_{c^{-}}$and $\mathbb{R}^{n}$-valued state components depend only on the time and input events yield (5). The computation of the $Q_{d}$-valued states along with checking Condition (2) - (3) is formalized in (6). Finally, Condition (4) is formalized in (7).

Theorem 3. The tuple $H_{\Delta}$ is a quasi-sequential transducer, and the sequential input-output map $R\left(H_{\Delta}\right)$ recognized by $H_{\Delta}$ is an abstraction of $R_{H}$.

\section{Finiteness of $\mathcal{R}(H)$ based on Lyapunov-like functions}

Theorem 4. Consider a finite set $\mathcal{X}_{0} \subseteq \mathcal{X}$ and a smooth map $V: \mathcal{X} \rightarrow \mathbb{R}$ such that for all $x \in \mathcal{X}, q=\left(q^{c}, q^{d}\right) \in Q$,

(1) $V(x) \geq 0$ and $V^{-1}(0) \subseteq \partial \mathcal{X}$.
(2) There exists $c>0$ such that $\operatorname{grad}(V)(x) f_{q^{c}}(x)<-c$, (3) For all $u \in E_{c}$, if $x \in \operatorname{int} \mathcal{X}$, then $V\left(R_{u, q}(x)\right) \leq V(x)$, and if $x \in \partial \mathcal{X}$, then $R_{u, q}(x) \in \mathcal{X}_{0}$.

It then follows that $\mathcal{R}(H)$ is finite.

Computation Notice that if the reset maps, flows of the vector fields (as in Definition 17), and the functions $h_{q, e}$ defining guards are (numerically) computable then so is $H_{\Delta}$. However, the computational complexity can get large as $\Delta$ decreases. Assumption 3. The reset maps of $H$ are affine in int $\mathcal{X}$, the vector fields are of L'ure-type, the state-space is a polyhedron, and the maps defining the guards are affine, i.e.

$$
\begin{aligned}
& \mathcal{X}=\left\{x \in \mathbb{R}^{n} \mid n_{i}^{T} x-b_{i} \leq 0, i=1,2, \ldots, K\right\} \\
& R_{u, q}(x)=M_{u, q} x+b_{u, q}, \quad \forall x \in \operatorname{int} \mathcal{X} \\
& h_{q, e}(x)=g_{q, e}^{T} x+d_{q, e}, \quad \forall x \in \mathbb{R}^{n} \\
& f_{q^{c}}(x)=A_{q^{c}} x+\sum_{j=1}^{m} B_{q^{c}, j} \phi_{q^{c}, j}\left(r_{q^{c}, j}^{T} x\right), \quad \forall x \in \mathbb{R}^{n} \\
& \mu_{1} \sigma+\gamma_{1} \leq \phi_{q^{c}, j}(\sigma) \leq \mu_{2} \sigma+\gamma_{2}, \quad \forall \sigma \in \mathbb{R}
\end{aligned}
$$

for matrices $M_{u, q}, A_{q^{c}} \in \mathbb{R}^{n \times n}$, vectors $b_{u, q}, r_{q^{c}, j}, B_{q^{c}, j}, g_{q, e}$, $n_{i} \in \mathbb{R}^{n}$, and scalars $d_{q, e}, b_{i}, \mu_{1}, \mu_{2}, \gamma_{1}, \gamma_{2} \in \mathbb{R}, q=$ $\left(q^{c}, q^{d}\right) \in Q, e \in E_{i} \cup E_{o}, u \in E_{c}, i=1,2, \ldots, K$, $j=1,2, \ldots, m$. The maps $\phi_{q^{c}, j}: \mathbb{R} \rightarrow \mathbb{R}, j=1,2, \ldots, m$ are piecewise-affine, continuous, globally Lipschitz.

If $H$ satisfies Assumption 3, then the reset maps and the maps $h_{q, e}$ are computable. The solution of $\dot{z}=f_{q^{c}}(z)$ can be computed using numerical integration. Hence, if we can detect reaching the boundary of $\mathcal{X}$, then the flow $f_{q^{c}}^{t}$ is computable. In fact, the definition of $H_{\Delta}$ can be modified so that it is enough to detect if the solution of $\dot{z}=f_{q^{c}}(z)$ has crossed the boundary, the precise point where the boundary was crossed is not needed. The latter is easy if the sign of each $n_{i}^{T} f_{q^{c}}(x)$, $i=1,2, \ldots, K$ is independent of $x$. Finiteness of $\mathcal{R}(H)$ can be checked effectively using Theorem 4 and the following.

Proposition 1. Assume that $H$ satisfies Assumption 3. If for some $j \in\{1, \ldots, K\}, c>0$, it holds that for all $x \in \mathcal{X}$,

(1) $n_{j}^{T}\left(A_{q^{c}} x+\sum_{l=1}^{m} \mu_{i_{l}}\left(B_{q^{c}, l} r_{q^{c}, l}^{T} x+\gamma_{i_{l}} B_{q^{c}, l}\right)\right)>c$, for any sequence $i_{1}, i_{2}, \ldots, i_{m} \in\{1,2\}$, and for any $q^{c} \in Q$,

(2) If $x \in \operatorname{int} \mathcal{X}$, then $n_{j}^{T}\left(M_{u, q} x-x+b_{u, q}\right) \geq 0$, for all $u \in E_{c}, q \in Q$

then $V(x)=\left(b_{j}-n_{j}^{T} x\right)$ satisfies Theorem 4 .

\section{ILLUSTRATING EXAMPLE}

Below we illustrate the theory by an example related to a control problem for printers from Petreczky et al. (2008b).

Formal model of the plant We will use the following parameters, meaning of which is described in Petreczky et al. (2008b): Fp, Cp, $\mathbf{V}_{\max }, \mathbf{V}_{\min }, \mathbf{T}_{f o}, \mathbf{T}_{p l, \max }, \mathbf{T}_{p l, \min }, \mathbf{A}, \mathbf{D}$. Formally, the plant model $H$ is of the form (3). The components of $H$ are explained below. The event sets are $E_{c}=$ $\left\{\mathbf{c}_{F U}, \mathbf{c}_{F D}, \mathbf{c}_{A}, \mathbf{c}_{D}\right\}, E_{o}=\left\{\mathbf{e}_{o, P L}\right\}, E_{d}=\left\{\mathbf{e}_{d, P L}\right\}, E_{i}=$ $\left\{\mathbf{e}_{N P I F}, \mathbf{e}_{i, P L}, \mathbf{e}_{\min , P L}, \mathbf{e}_{\max , P L}, \mathbf{e}_{F U c}\right\}$. The discrete statespace $Q=Q_{c} \times Q_{d}$ is defined as follows. $Q_{d}$ is the set of maps $\phi: \operatorname{Var}_{d} \rightarrow\{$ True, False $\}$, where $\operatorname{Var}_{d}=$ $\left\{\mathbf{S}_{P L}, \mathbf{S}_{r}, \mathbf{S}_{F U c}\right\} . Q_{c}$ is the set of all maps $\phi: \operatorname{Var} \rightarrow$ $\{$ True, False $\}$ where $\operatorname{Var}=\left\{\mathbf{S}_{F U}, \mathbf{S}_{F D}, \mathbf{S}_{A}, \mathbf{S}_{D}\right\}$. I.e. the elements of $Q_{d}$ and $Q_{c}$ are valuations of predicates from $\operatorname{Var}_{d}$ and $\operatorname{Var}_{c}$ respectively. In the sequel, we will write $\phi(X)$ instead of $\phi(X)=$ True, and $\neg \phi(X)$, instead of $\phi(X)=$ False for all $\phi \in Q_{d}, X \in \operatorname{Var}_{d}$, or $\phi \in Q_{c}$ and $X \in \operatorname{Var}_{c}$. The continuous state-space is $\mathcal{X}=\left\{x=\left(\mathbf{P}, \mathbf{V}, \mathbf{C}_{f u}, \mathbf{T}\right) \in \mathbb{R}^{4}\right.$ $\mathbf{P} \leq \mathbf{C p}\}$ where $\mathbf{P}, \mathbf{V}, \mathbf{C}_{f u}, \mathbf{T} \in \mathbb{R}$ are state variables. The 
vector fields $f_{q^{c}}, q^{c} \in Q_{c}$ and the reset maps $R_{u, q}, q \in Q$, $u \in E_{c}$ are as follows. For any $x=\left(\mathbf{P}, \mathbf{V}, \mathbf{C}_{f u}, \mathbf{T}\right) \in \mathcal{X}$,

$$
\begin{aligned}
& f_{q^{c}}(x)=\left[\max \left\{\mathbf{V}_{\text {min }}, \mathbf{V}\right\} f_{2, q^{c}}(x) \quad 1 \quad 1\right]^{T} \\
& f_{2, q^{c}}(x)= \begin{cases}\mathbf{A} \phi_{\min }(x) \phi_{\max }(x) & \text { if } q^{c}\left(\mathbf{S}_{A}\right)\end{cases} \\
& \left\{-\mathbf{D} \phi_{\min }(x) \phi_{\max }(x) \text { if } q^{c}\left(\mathbf{S}_{D}\right) \text { and } q^{c}\left(\mathbf{S}_{F D}\right)\right. \\
& \phi_{\min }(x)= \begin{cases}1 & \text { if } \mathbf{V} \in\left(\mathbf{V}_{\text {min }}+\epsilon,+\infty\right) \\
\frac{\left(\mathbf{V}-\mathbf{V}_{\text {min }}\right)}{\epsilon} & \text { if } \mathbf{V} \in\left(\mathbf{V}_{\text {min }}, \mathbf{V}_{\text {min }}+\epsilon\right] \\
0 & \text { if } \mathbf{V} \in\left(-\infty, \mathbf{V}_{\text {min }}\right]\end{cases} \\
& \phi_{\max }(x)= \begin{cases}1 & \text { if } \mathbf{V} \in\left(-\infty, \mathbf{V}_{\max }-\epsilon\right) \\
\underline{\left(\mathbf{V}_{\max }-\mathbf{V}\right)} & \text { if } \mathbf{V} \in\left[\mathbf{V}_{\max }-\epsilon, \mathbf{V}_{\max }\right) \\
0 & \text { if } \mathbf{V} \in\left[\mathbf{V}_{\max },+\infty\right)\end{cases} \\
& R_{u, q}(x)= \begin{cases}(\mathbf{P}, \mathbf{V}, 0, \mathbf{T}) & \text { if } u=\mathbf{c}_{F D} \text { and } \mathbf{P}<\mathbf{C p} \\
\left(\mathbf{P}, \mathbf{V}, \mathbf{C}_{f u}, \mathbf{T}\right) & \text { if } u \neq \mathbf{c}_{F D} \text { and } \mathbf{P}<\mathbf{C p} \\
\left(\mathbf{C p}, \mathbf{V}_{\text {max }}, \mathbf{T}_{f o}, \mathbf{T}_{p l, \text { max }}\right) & \text { if } \mathbf{P}=\mathbf{C p}\end{cases}
\end{aligned}
$$

The state-transition maps $\delta_{c}$ and $\delta_{d}$ are such that for each $q_{1}=\left(q_{1}^{c}, q_{1}^{d}\right) \in Q, u \in E_{c}, e \in E_{i} \cup E_{d}, \delta_{c}\left(q_{1}, u\right)=q_{2}^{c}$ and $\delta_{d}\left(q_{1}, e\right)=q_{2}^{d}$ if and only if the following holds.

$$
\begin{aligned}
& \left(q_{2}^{c}\left(\mathbf{S}_{F D}\right), q_{2}^{c}\left(\mathbf{S}_{F U}\right)\right)= \begin{cases}(\text { True }, \text { False }) & \text { if } u=\mathbf{c}_{F D} \\
(\text { False }, \text { True }) & \text { if } u=\mathbf{c}_{F U} \\
\left(q_{1}^{c}\left(\mathbf{S}_{F D}\right), q_{1}^{c}\left(\mathbf{S}_{F U}\right)\right) & \text { otherwise }\end{cases} \\
& \left(q_{2}^{c}\left(\mathbf{S}_{A}\right), q_{2}^{c}\left(\mathbf{S}_{D}\right)= \begin{cases}(\text { False }, \text { True }) & \text { if } u=\mathbf{c}_{D} \\
(\text { True } \text { False }) & \text { if } u=\mathbf{c}_{A} \\
\left(q_{1}^{c}\left(\mathbf{S}_{A}\right), q_{1}^{c}\left(\mathbf{S}_{D}\right)\right) & \text { otherwise }\end{cases} \right. \\
& q_{2}^{d}\left(\mathbf{S}_{P L}\right)=\left\{\begin{aligned}
\text { True } & \text { if } e=\mathbf{e}_{d, P L} \text { and } q_{1}^{d}\left(\mathbf{S}_{r}\right) \\
q_{1}^{d}\left(\mathbf{S}_{P L}\right) & \text { otherwise }
\end{aligned}\right. \\
& q_{2}^{d}\left(\mathbf{S}_{r}\right)=\left\{\begin{aligned}
\text { True } & \text { if } e=\mathbf{e}_{\min , P L} \text { and } \neg q_{1}^{d}\left(\mathbf{S}_{r}\right) \\
\text { False } & \text { if } e=\mathbf{e}_{\max , P L} \text { and } q_{1}^{d}\left(\mathbf{S}_{r}\right) \\
q_{1}^{d}\left(\mathbf{S}_{r}\right) & \text { otherwise }
\end{aligned}\right. \\
& q_{2}^{d}\left(\mathbf{S}_{F U c}\right)=\left\{\begin{aligned}
\text { True } & \text { if } e=\mathbf{e}_{F U c} \\
q_{1}^{d}\left(\mathbf{S}_{F U c}\right) & \text { otherwise }
\end{aligned}\right.
\end{aligned}
$$

The readout maps $\lambda_{o}$ and $\lambda_{i}$ are defined as follows; $\lambda_{i}\left(q, e_{d}\right)=$ $\mathbf{e}_{i, P L}$ and $\lambda_{o}\left(q, e_{d}\right)=\mathbf{e}_{o, P L}$. The guard are defined as follows. $\Phi_{q, e} \subseteq\left\{x \in \operatorname{int} \mathcal{X} \mid h_{q, e}(x)=0\right\}, \forall e \in\left(E_{i} \cup E_{o}\right) \backslash\left\{\mathbf{e}_{o, P L}, \mathbf{e}_{i, P L}\right\}$,

$\Phi_{q, \mathbf{e}_{o, P L}}=\Phi_{q, \mathbf{e}_{i, P L}}=\emptyset$ and $\Phi_{q, e_{1}} \cap \Phi_{q, e_{2}}=\emptyset, \forall e_{1} \neq e_{2} \in E_{i}$

$h_{q, \mathbf{e}_{F U c}}(x)= \begin{cases}\left(x_{3}-\mathbf{T}_{f o}\right\} & \text { if } q^{c}\left(\mathbf{c}_{F U}\right) \\ 1 & \text { otherwise }\end{cases}$

$h_{q, \mathbf{e}_{\text {min }, P L}}(x)= \begin{cases}\left(x_{4}-\mathbf{T}_{p l, m i n}\right) & \text { if } \neg q^{d}\left(\mathbf{S}_{r}\right) \text { and } \neg q^{d}\left(\mathbf{S}_{P L}\right) \\ 1 & \text { otherwise }\end{cases}$

$h_{q, \mathbf{e}_{\max , P L}}(x)= \begin{cases}\left(x_{4}-\mathbf{T}_{p l, \max }\right) & \text { if } \neg q^{d}\left(\mathbf{S}_{P L}\right) \text { and } q^{d}\left(\mathbf{S}_{r}\right) \\ 1 & \text { otherwise }\end{cases}$

$h_{q, \mathbf{e}_{N P I F}}(x)= \begin{cases}x_{1}-\mathbf{F p} & \text { if } q^{d}\left(\mathbf{S}_{P L}\right) \text { and } \\ & \left(q^{c}\left(\mathbf{S}_{F D}\right) \text { or }\left(q^{c}\left(\mathbf{S}_{F U}\right) \text { and } \neg q^{d}\left(\mathbf{S}_{F U c}\right)\right)\right) \\ 1 & \text { otherwise }\end{cases}$

The initial state $h_{0}=\left(q_{0}^{c}, q_{0}^{d}, x_{0}\right)$ is of the following form.

$$
\begin{aligned}
& q_{0}^{c}(X)=\text { False }, X \in \mathbf{V a r}_{c} \backslash\left\{\mathbf{S}_{F D}\right) \text { and } q_{0}^{c}\left(\mathbf{S}_{F D}\right)=\text { True } \\
& q^{d}(Y)=\text { False, } \forall Y \in \mathbf{V a r}_{d} \text { and } x_{0}=\left(0, \mathbf{V}_{\text {max }}, 0,0\right)
\end{aligned}
$$

Control requirements $K=\left(E_{i} \backslash \mathbf{e}_{N P I F}\right)^{*} \cup\left(E_{i} \backslash \mathbf{e}_{N P I F}\right)^{\omega}$ Solution It is easy to see that Assumption A.1- A.3 and Assumption 3 are satisfied for $H$. We can solve Problem 1 for $H$ and $K$ above using the procedure outlined in $\S 4$. Notice that $H_{\Delta}$ is computable, and $\mathcal{R}(H)$ is finite. For the latter, define $\mathcal{X}_{0}=\left\{\left(\mathbf{C p}, \mathbf{V}_{\max }, \mathbf{T}_{f o}, \mathbf{T}_{p l, \max }\right)\right\}$, and define the $\operatorname{map} V: \mathcal{X} \rightarrow \mathbb{R}$ as $V\left(x_{1}, x_{2}, x_{3}, x_{4}\right)=\left(\mathbf{C p}-x_{1}\right)$. It follows from Proposition 1 that $V$ and $\mathcal{X}_{0}$ satisfy Theorem 4. In Petreczky et al. (2008b) controllers were synthesized based on an algorithm and a model related to the one presented above.

\section{DISCUSSION AND CONCLUSIONS}

We have presented a control problem for a class of hybrid systems and we have proposed a solution based on computing finite-state discrete-event abstraction of hybrid systems. We believe that the results are relevant for practice. Future research includes extension of the results to other classes of systems and the study of robustness and computational issues.

\section{REFERENCES}

Alur, R., Henzinger, T., Lafferriere, G., and Pappas, G.J. (2000). Discrete abstractions of hybrid systems. Proccedings of the IEEE, 88(2), 971-984.

Belta, C., Isler, V., and Pappas, G. (2005). Discrete abstractions for robot motion planning and control in polygonal environments. IEEE Transactions on Robotics, 21(5), 864-874.

Berstel, J. (1979). Transductions and Context-Free Languages. Teubner, Stuttgart.

Chutinan, A. and Krogh, B.H. (2003). Computational techniques for hybrid system verification. IEEE Trans. Automatic Control, 48(1).

Eilenberg, S. (1974). Automata, Languages and Machines. Academic Press, New York, London.

Fainekos, G.E., Girard, A., and Pappas, G.J. (2007). Hierarchical synthesis of hybrid controllers from temporal logic specifications. In HSCC, 203-216.

Förstnera, D., Jung, M., and Lunze, J. (2002). A discrete-event model of asynchronous quantised systems. Automatica, 38, $1277-1286$.

Gonzalez, J., da Cunha, A., Cury, J., and Krogh, B. (2001). Supervision of event-driven hybrid systems: Modeling and synthesis. In Hybrid Systems: Computation and Control, volume LNCS 2034, $247-260$.

Grädel, E., Thomas, W., and Wilke, T. (2002). Automata, Logic and Infinite Games, volume LNCS 2500. Springer.

Koutsoukos, X., Antsaklis, P., Stiver, J., and Lemmon, M. (2000). Supervisory control of hybrid systems. Proceedings of the IEEE.

Moor, T. and Raisch, J. (1999). Supervisory control of hybrid systems within a behavioural framework. Systems and Control Letters, 38, 157 - 166.

Moor, T., Raisch, J., and O'Young, S. (2002). Discrete supervisory control of hybrid systems based on l-complete approximations. Discrete Event Dynamic Systems, 12(1), 83-107.

Petreczky, M., Theunissen, R., Su, R., van Beek, D., van Schuppen J.H., and Rooda, J. (2008a). Control of input-output discrete-event systems. Technical Report 2008-12, Eindhoven University of Technology, Systems Engineering.

Petreczky, M., van Beek, D.A., and Rooda, J.E. (2008b). Supervisor for toner error-handling. Technical Report 2008-11, Eindhoven University of Technology, Systems Engineering.

Philips, P., Heemels, W., Preisig, H., and van den Bosch, P. (2003). Control of continuous-time quantised systems. Int. J. of Control, 76, 277-294.

Raisch, J. (2000). Discrete abstractions of continuous systems - an input/output point of view. Mathematical and Computer Modelling of Dynamical Systems, 6(1), 6-29.

Raisch, J. and O'Young, S. (1995). A des approach to control of hybrid dynamical systems. In Hybrid Systems, 563-574.

Tabuada, P. and Pappas, G.J. (2005). Hierarchical trajectory generation for a class of nonlinear systems. Automatica, 41(4), 701-708.

van der Schaft, A. and Schumacher, H. (2000). An Introduction to Hybrid Dynamical Systems. Springer-Verlag London. 